\title{
Frontières
}

\section{Libera me domine de morte}

L'organisation d'un rituel liturgico-musical de la mort et

l'exemple du ms. Allbi, B.M 15 (XI ${ }^{\mathrm{e}}$ siècle)

\section{Olivier Cullin}

Volume 20, numéro 2, printemps 2008

Les musiques et la mort

URI : https://id.erudit.org/iderudit/018329ar

DOI : https://doi.org/10.7202/018329ar

Aller au sommaire du numéro

Éditeur(s)

Université du Québec à Montréal

ISSN

1180-3479 (imprimé)

1916-0976 (numérique)

Découvrir la revue

Citer cet article

Cullin, O. (2008). Libera me domine de morte : l'organisation d'un rituel liturgico-musical de la mort et l'exemple du ms. Albi, B.M 15 (XI ${ }^{\mathrm{e}}$ siècle). Frontières, 20(2), 22-25. https://doi.org/10.7202/018329ar
Résumé de l'article

La liturgie médiévale de la mort est souvent restée marginale, conservant ainsi une organisation complexe et locale. L'exemple du rituel conservé dans le ms. d'Albi, B.M 15 ( $\mathrm{xi}^{\mathrm{e}}$ siècle) est caractéristique des variations et interprétations que le Moyen Âge a apportées sur la mort, le défunt lui-même et la promesse d'une vie éternelle. L'étude précise de deux répons originaux montrera comment le rituel tient lieu d'intercession entre un mort qui parle encore et la communauté des vivants, comment s'organise les thèmes apocalyptiques avec l'idée d'une salvation promise. 


\section{Résumé}

La liturgie médiévale de la mort est souvent restée marginale, conservant ainsi une organisation complexe et locale. L'exemple du rituel conservé dans le ms. d'Albi, B.M 15 ( $\mathrm{Xl}^{\mathrm{e}}$ siècle) est caractéristique des variations et interprétations que le Moyen Âge a apportées sur la mort, le défunt lui-même et la promesse d'une vie éternelle. L'étude précise de deux répons originaux montrera comment le rituel tient lieu d'intercession entre un mort qui parle encore et la communauté des vivants, comment s'organise les thèmes apocalyptiques avec l'idée d'une salvation promise.

Mots clés: musique - liturgie - Moyen Âge - rituel.

\begin{abstract}
Medieval liturgy of the Dead has often been marginal, keeping in itself a complex and local organization. The example of the ritual in Albi B.M 15 (XIth century) is revealant of variations and interpretations on medieval approach of death, the defunct and the promise of an eternal life. This study of two precise and original responsories will show how the ritual is an intercession between a speaking defunct and the whole living community, how are organized apocalyptic ideas with the promised redemption.
\end{abstract}

Keywords: music - liturgy Middle Ages - ritual.

\section{Libera me domine de morte}

\section{L'organisation d'un rituel liturgico-musical de la mort et l'exemple du ms. Albi, B.M 15 (Xle siècle)}

\author{
Olivier Cullin, \\ professeur, CESCM, CNRS UMR 6223 , \\ Université de Poitiers.
}

Comme toutes les sociétés, la société médiévale a entouré d'honneur et de vénération les dépouilles des défunts. Dès le $I^{\mathrm{e}}$ siècle, il existe un office complet des défunts dans la liturgie romaine et, au Moyen Âge, cet office est en usage dans presque toutes les églises. Il comprend les Vêpres, les Matines et les Laudes puis la messe des défunts. Cet ensemble liturgique et musical tient néanmoins une place particulière en permettant, à partir d'une trame liturgique commune, de nombreuses adaptations locales ${ }^{1}$ ou bien en étant traditionnellement situé dans les sources manuscrites en position marginale: dans les manuscrits liturgiques, la messe des défunts se trouve à la fin, parmi d'autres messes particulières comme celle de la Dédicace. La situation marginale de cet office est justement celle du ms. Albi 15 (152). Ce livre est un lectionnaire du $\mathrm{XI}^{\mathrm{e}}$ siècle qui contient, à partir du fo 164 , un cahier final ajouté et noté en notation aquitaine dans lequel figure notamment la liturgie des morts, présentée dans l'ordre suivant:

- $f^{\circ} 164 r-165 v$ : répons des nocturnes des Matines

- $\mathrm{f}^{\circ}$ 166r-v: antiennes des Matines et des Laudes

- $\mathrm{f}^{\circ} 166 \mathrm{v}$ : office des Vêpres ${ }^{2}$

Par ailleurs, entre le $\mathrm{IX}^{\mathrm{e}}$ siècle où l'office commence à se généraliser et la fin $d u X{ }^{\mathrm{e}}$ siècle qui voit l'apparition du Purgatoire, l'office des défunts marque une évolution progressive dans le concept d'une vie après la mort. La mort est d'abord per- çue comme un long sommeil dans l'attente du retour du Seigneur. Avec le développement des thèmes apocalyptiques et du Jugement dernier, la mort est l'étape à partir de laquelle l'âme du défunt, après avoir passé le premier jugement, est soumise à une sorte de purification à laquelle répondent, pour les vivants, l'office et les prières comme un rituel de tempérance. Enfin, le Purgatoire introduit clairement cette transition entre l'Enfer et le Paradis (Le Goff, 1981). Les lectures tirées du Livre de Job transportées de la veillée mortuaire aux leçons de l'office des matines prennent une signification particulière. Les lamentations de Job n'ont pas, en effet, de rapport direct avec la mort physique, mais encloses dans le rituel mortuaire, elles transportent avec elles la plainte et surtout la souffrance de l'âme dans l'attente de la vraie vie. L'étude portera donc sur l'office des matines qui se distingue, dans Albi 15, par un répertoire original. Il conviendra d'interroger sa place et son sens par rapport à l'évolution théologique et spirituelle du concept d'une vie après la mort, un thème qui prend au Moyen Âge de longs et sinueux développements.

L'office est habituellement composé de trois nocturnes comprenant chacun trois antiennes, trois leçons (lecture), trois répons avec leurs versets intercalés dans les leçons. La répartition des répons dans les trois nocturnes formant l'office est la suivante :

$1^{\mathrm{er}}$ nocturne

$[14]^{3}$ R. Credo quod redemptor

Je crois que le Rédempteur

V. Quem visurus sum [177]

Que je vais voir 
[72] R. Qui Lazarum resuscitasti

Toi qui as ressuscité Lazare

V. Requiem eternam [198]

[Donne] le repos éternel

[82] R. Requiem eternam

[Donne] le repos éternel

V. Anime eorum [15]

Leurs âmes

$2^{\mathrm{e}}$ nocturne

[24] R. Domine dum veneris

Seigneur quand tu viendras

V. Delicta mea [47]

Mes fautes

[32] R. Heu mihi domine

Hélas pour moi, Seigneur

V. Convertere domine [35]

Convertis, Seigneur

[57] R. Ne recorderis peccata mea Ne te souviens pas de mes péchés

V. Tibi soli peccavi [222]

J'ai péché seul contre toi

$3^{\text {e }}$ nocturne

[68] R. Peccantem me cotidie

En péchant chaque jour

V. Deus in nomine tuo [53]

Seigneur en ton nom

[56] R. Ne perdas me domine

Ne me perds pas Seigneur

$\mathrm{V}$. Ne intres in judicio [152]

Ne me juges pas

[40] R. Libera me domine de viis

Libère moi, Seigneur, des chemins

[de l'enfer]

V. Clamantes et dicentes [31]

En s'exclamant et disant

V. Claustra inferni confracta sunt

[30]

Les chaînes de l'enfer sont brisées

Cet enchaînement 14-72-82 / 24-32-47

/ 68-56-40 constitue une typologie propre parmi d'autres possibilités ${ }^{4}$. L'ensemble de l'office est récité in persona defuncti (Martimort, 1961, p. 624-625) et les textes expriment les sentiments d'un mort qui, en fait, ne l'est pas : il crie et se lamente; il affirme son espérance (R. 14 et 24). Dans le même temps, certains répons changent de locuteur et s'affirment davantage comme une prière pour le mort (R. 72). L'office oscille donc toujours entre l'expression singulière du mort et celle collective de la communauté intercédant pour lui : cette oscillation donnera lieu, comme nous le verrons, à des aménagements textuels conscients dans Albi 15. Le premier nocturne souligne, avec insistance ici, la demande de paix : au verset Requiem eternam (198) succède le répons (198) sur le même texte. Le deuxième nocturne présente un enchaînement dont le ms. Albi 15 est le plus ancien témoin connu ${ }^{5}$. La troisième section est, elle aussi, assez originale dans son enchaînement: on la retrouve dans des sources plus tardives, à Toulouse



Marie-Madeleine, détail de Lamentation sur le corps du Christ, dernier quart du XV ${ }^{\mathrm{e}}$ siècle, Santa Maria della Vita, Bologne.

notamment (Ottosen, 1993, p. 169). Le dernier répons ajoute un verset supplémentaire Claustra inferni évoquant le moment où les chaînes de l'enfer se brisent, les tombes des saints s'ouvrent et les voix se réunissent unanimement dans l'attente du Dieu roi ${ }^{6}$. Ce verset est rare et ne figure pas dans le Corpus Antiphonalium Officii de Dom Hesbert. Il n'existe, dans cet état, que dans Albi 15 même si on retrouve le même incipit ensuite dans deux sources postérieures de Braga (Portugal) et Oviedo (Espagne $)^{7}$.

À cet ensemble liturgique succède, dans le manuscrit, une liste supplétive exceptionnelle de cinq répons ad libitum comprenant, pour le premier, une suite remarquable de versets:

[38] R. Libera me domine de morte

Libère moi Seigneur de la mort

V. Tremens factus sum [227]

Je suis tremblant

V. Dies illa, dies ira [55]

Ce jour là, jour de colère

V. Plangent se [178]

[Les peuples de la terre] se lamentent

V. Quid ergo miserrimus [182]

Quoi donc très misérable

V. Nunc Christe te deprecor [159]

Maintenant, Christ, je te supplie

V. Et voces archangeli [422]

Et les voix des archanges

V. Jesu redemptor miserere* [399]

Jésus rédempteur, aies pitié

V. Ingemiscite super me* [259]

Lamentez vous sur moi

V. Creator omnium rerum [38]
Créateur de toutes choses

V. Dies miseriarum [437]

Jour de malheur

V. Graviter mala* [115]

Les péchés [m’ont enlacé] lourdement

V. Heu me! Miser, infelix* [103]

Hélas pour moi! Misérable

et malheureux

V. Jesu domine qui venisti* ${ }^{[111]}$

Jésus Seigneur, toi qui es venu

V. Qualis pavor [438]

De quel effroi

V. Tremor magnus* [228]

Un grand tremblement

V. Lux immarcescibilis* [133]

Une lumière éclatante

V. Vox venit de celo [439]

Une voix est venue du ciel

V. Celum et terra [440]

Le ciel et la terre

V. Jesu clementer [110]

Jésus très clément

V. Ibi dolor, ibi luctus [447]

Là où est la douleur, là est

le chagrin

V. Redemptor piissime ${ }^{*}$ [302]

Rédempteur très pieux

V. Hoc enim vobis [441]

En vérité, à vous [nous disons]

V. O viri Israelite [442]

Ô, peuple d'Israël

[R. Libera me]

V. Conditor et redemptor [443]

Créateur et Rédempteur

V. Ad quem factura tua [444]

Tes bienfaits [Seigneur] à qui [irontils]

V. Qui super astra sedis [445]

Toi qui trônes sur les astres

V. Tu dixisti domine* [253]

$\mathrm{Tu}$ as dit Seigneur

V. Mortis mors* [392]

La mort de la mort

V. Tu me pro dolor [446]

Toi, pour la douleur

V. Parce fili dei* [322]

Pardonne, fils de Dieu

V. Requiem eternam [198]

[Donne] le repos éternel

[50] R. Mundi redemptor domine

Seigneur, rédempteur du monde

V. Ubi resultant organa angelorum [231]

Là ou résonnent les chants des anges

V. Qui decantant [279]

Qui chantent ensemble

V. Cherubim ardentia [255]

Les chérubins ardents

V. Canendo tibi [24]

Chantant pour toi

V. Cum quibus nunc per omnia [42]

Avec lesquels, maintenant et pour toujours 
V. Et tu rex seculorum suscipe [200]

Et toi, roi de tous les temps, accueille

V. Eternam requiem dona eis [89]

Donne lui le repos éternel

[1] R. Absolve

V. Si que sunt domine [216]

[79] R. Redemptor meus

V. Lauda anima [125]

[83] R. Rogamus te domine 8

Le cas du répons Libera me est évidemment très intéressant. Dans de très nombreuses sources, il est le dernier répons $\mathrm{du}$ troisième nocturne, soit à la place du répons Libera me... de viis, soit en lui succédant immédiatement. Il est très souvent proposé comme répons supplémentaire, en position de substitution avec ce même répons. La version d'Albi 15 est particulièrement impressionnante. Elle s'ouvre sur les cinq versets les plus communément diffusés formant la base même du répons (V. 227, 55, 178, 182, 159 et 38). Un second groupe lui est adjoint qui sera diffusé par la suite dans d'autre sources mais pour lequel Albi 15 est le premier témoin connu (versets signalés par un astérisque: V. 399, 259, 103, 111, 228, 133, 302, 253, 392 et 322). Enfin, un troisième groupe complètement inédit de douze versets est inséré dans cet ensemble (versets en gras: V. 422, 437447). La structure complexe de ce répons présente donc, dans ces trois groupes qui le forment, un programme eschatologique remarquable de trois niveaux de sens imbriqués les uns dans les autres.

Le premier ensemble décrit les plaintes et les craintes du Jugement dernier mais, aux interrogations sur la salvation promise contenues normalement dans le verset Plangent, on a préféré adoucir l'effroi en introduisant très vite le pardon et la salvation et en intervertissant cette fin avec celle du verset Nunc Christe te deprecor ${ }^{9}$. Cette construction particulière, si elle n'évacue pas l'angoisse du jugement, la place à un double niveau: celui d'une attente préalablement rappelée avant que ne soit nommé le Christ juge, et celui d'une expression collective («les tribus de la terre»). Elle introduit plus fortement ensuite la relation personnelle entre un Christ juge, mais dont on a dit qu'il pardonne, et le défunt qui parle à la première personne ${ }^{10}$.

Le deuxième groupe de versets prône la nécessité d'une clémence divine universelle (V. 259, 133), seul remède aux tourments (V. 115, 228), et oppose au pardon (V. 399, 392) la situation d'affliction et de misère dans lequel le défunt, parlant toujours à la première personne, se trouve (V. 103). La consolation est évoquée (V. 111) ainsi que la promesse de la vie éternelle pour ceux qui auront cru (V. 253, 302, 322).

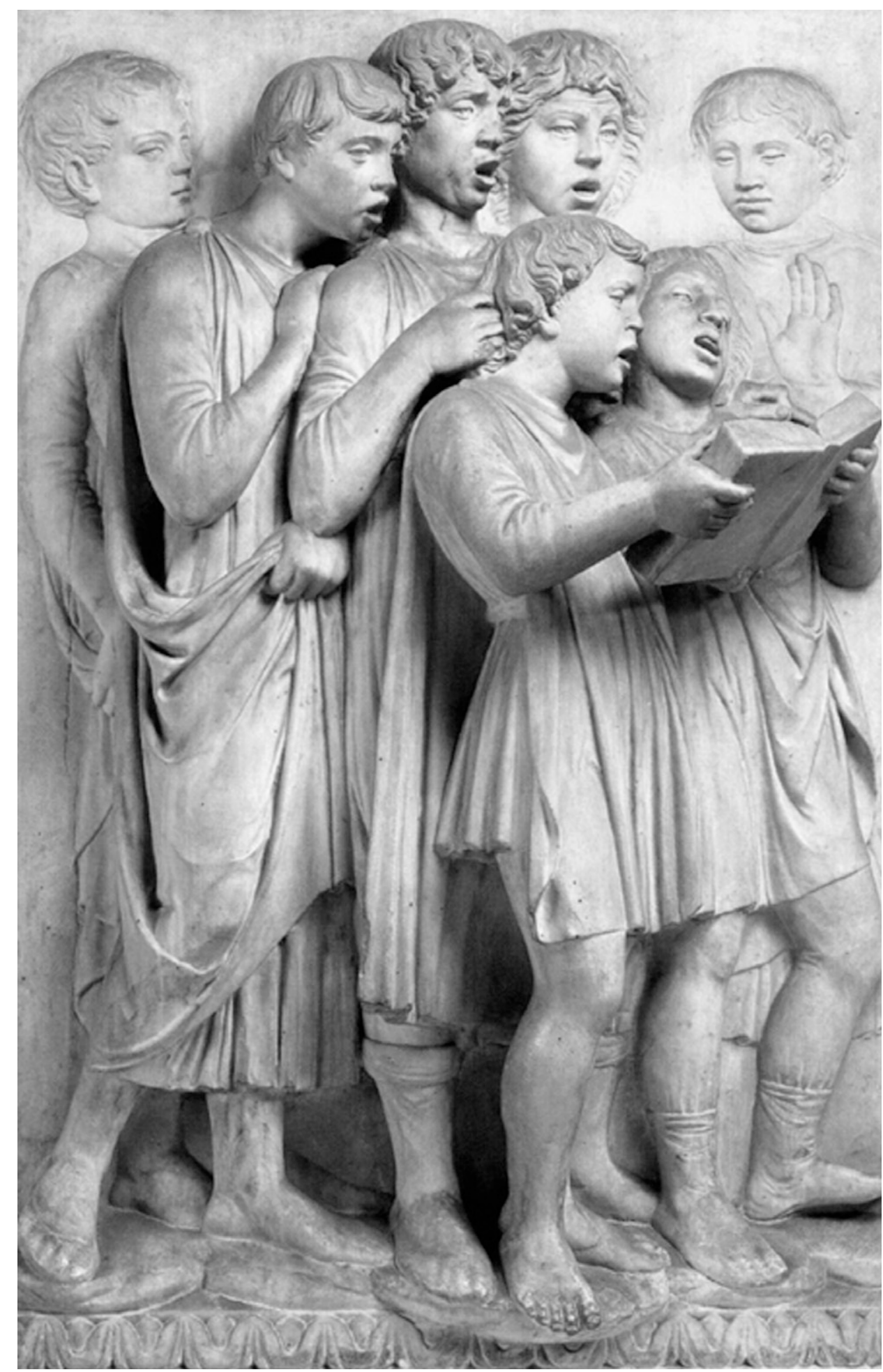

Luca della ROBBIA, Cantoria, 1431-38, Marbre, $328 \times 560 \mathrm{~cm}$, Museo dell'Opera del Duomo, Florence.

Le troisième et dernier groupe est propre à Albi 15. Il est exclusivement organisé autour du thème du Jugement dernier dont il développe le programme: les trompettes du jugement (V. 422), le jour de fureur (V. 437), les enfers horribles qui s'ouvrent (V. 438), la voix qui convoque les morts (V. 439), la confusion du ciel et de la terre (V. 440), la venue du Christ (V. 441), siégeant à la droite du Père (V. 445), pour juger (V. 446).
Le répons suivant Mundi redemptor est une création $\mathrm{du} \mathrm{XI}^{\mathrm{e}}$ siècle dont la diffusion est extrêmement limitée. On ne le rencontre que dans quatre sources connues - trois manuscrits italiens et Albi 15 (Ottosen, 1993, p. 302-303; 318) ${ }^{11}$. Le thème du répons fait suite à la vision apocalyptique de la pièce précédente et déroule la vision angélique où l'armée des anges, des séraphins et des chérubins, à la droite du Père, porte l'âme du défunt 
au Paradis et, par des cantiques, chante la gloire éternelle du Père (V. 231, 279, $55,24)$. La composition de ces versets est particulière et totalement en prise avec la réalité esthétique $\mathrm{du} \mathrm{XI}{ }^{\mathrm{e}}$ siècle. Il s'agit, en effet, d'une composition dont la poétique, les éléments formels, les images, le style musical prennent leur inspiration dans le style des nova cantica, ces compositions poétiques et musicales nouvelles qui enrichirent à travers les proses, les tropes et les séquences, la liturgie romane à partir $\mathrm{du} \mathrm{X}^{\mathrm{e}}$ siècle. On est donc, ici, en prise avec une réalité qui développe une vision particulière du Jugement dernier (la vision béatifique) dans une expression adaptée à la sensibilité d'une époque. Chaque verset, à l'exception du dernier Requiem eternam qui n'est pas propre à cette composition, suit une structure métrique, assonancée et rimée qui l'apparente au schéma d'une séquence:

V. 231 Ubi resultant organa

Là où résonnent les chants angelorum melliflua des anges coulant comme du miel,

V. 279 Qui decantant frequentia Là chantent en foule alti celi palatia dans le palais des cieux infinis

V. 255 Cherubin ardencia les chérubins ardents et seraphin altitona les séraphins dans tous les tons

V. 24 Canendo tibi cantica En chantant des cantiques pour toi Sedenti patri dextera qui es assis à la droite du Père

V. 42 Cum quibus nunc per omnia Avec lesquels, maintenant et pour toujours tripudiet per secula [le défunt] dansera

$8 \mathrm{a}$

$8 \mathrm{a}$ $8 a$

\section{$8 \mathrm{a}$} $7 a$

V. 200 Et tu rex seculorum tuorum 10b Et toi, roi de tous les temps, suscipe preces servorum $8 b$ reçois la prière de tes serviteurs

Le vocabulaire employé est très proche de celui que l'on rencontre dans les tropes du Sanctus, hymne angélique par excellence: l'énumération des ordres célestes, la référence au chant éternel des anges devant le trône de majesté («ici les chants des anges se répercutent») avec des termes précis (organa, cantica), l'idée d'un chant merveilleux, "coulant comme du miel » (melliflua) (voir Iversen, 2001). L'épithète melliflua est traditionnellement lié à la naissance du Christ dans l'expression caeli melliflui et, comme l'écrit Lars Elfving (Elfving, 1962), "la même pensée que le miel coule à la naissance d'un dieu se rencontre dans la poésie profane de l'Antiquité, par exemple dans la quatrième églogue de Virgile»(Elfving, 1962, p. 67-69).
Enfin, la musique est originale et reprend un seul et même timbre pour l'ensemble des versets, suivant encore en cela l'esprit d'une séquence et non le cheminement mélodique habituel d'un répons grégorien. Les auteurs de ce répons ont donc souhaité marquer le caractère théologique particulier (le jugement dernier débouchant sur la vision du chant angélique) par une composition esthétique singulière, neuve: ils ont fait coïncider l'actualité d'une thématique avec les cadres d'expression artistique contemporains.

La mort n'est pas une fin. Si aucun des textes contenus dans Albi 15 ne permet d'anticiper sur la thématique du Purgatoire qui se développera au XII ${ }^{\mathrm{e}}$ siècle, les remarques que nous avons données sur l'office des défunts ne le réduisent pas non plus en une antinomie manichéenne entre le ciel et l'enfer. La célébration de l'office sous-entend de manière tacite que les âmes, après la séparation du corps et avant le Jugement dernier, expérimentent une première "vie après la mort», remplie de crainte, de tourments mais aussi d'espérance. Les dispositions particulières rencontrées dans Albi 15 expriment clairement le double rôle de l'office et du chant, à la fois comme expression du mort qui parle en son nom propre et comme intercession collective laissée aux mains des vivants. Dans les deux répons étudiés, ces

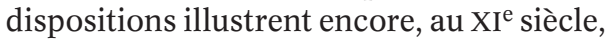
le renforcement puissant des thèmes apocalyptiques et du Jugement dernier mais en le liant à une rémission préalablement établie et en unissant la prière chantée des vivants à la musique éternelle des anges devant la majesté divine.

\section{Bibliographie}

CORBIN, S. (1952). Essai sur la musique religieuse portugaise au Moyen Âge (1100-1385), Paris, Les Belles Lettres.

CULLIN, O. (1982). "Une pièce gallicane conservée par la liturgie de Gaillac. L'offertoire Salvator mundi », Cahiers de Fanjeaux, $\mathrm{n}^{\circ} 17$, p. 287-297.

DESACHY, M. (2007). Le scriptorium d'Albi. Les manuscrits de la cathédrale Sainte-Cécile (VII ${ }^{e}-X_{I}^{e}$ siècles), Rodez, Éditions du Rouergue.

ELFVING, L. (1962). Étude lexicographique sur les séquences limousines, Stockholm (Suède), Almqvist \& Wiksell.

IVERSEN, G. (2001). Chanter avec les anges, Paris, Cerf.

LE GOFF, J. (1981). La naissance du Purgatoire, Paris, Gallimard.

MARTIMORT, A.-G. (1961). L'Église en prière. Introduction à la liturgie, Paris, Desclée.
OTTOSEN, K. (1993). The Responsories and Versicles of the Latin Office of the Dead, Aarhus (Danemark), Aarhus University Press.

\section{Notes}

1. Comme le montre fort bien le catalogue thématique édité par Knud Ottosen (1993). J'ai moi-même observé ces « originalités » locales dans une source proche d'Albi 15 (voir Cullin, 1982).

2. Après le $\mathrm{f}^{\circ} 166 \mathrm{v}$, le manuscrit contient l'office de la translation de Lazare et l'office de saint Baude.

3. Les numéros entre crochets renvoient aux numéros de la pièce dans le catalogue thématique de Knud Ottosen (1993).

4. Je renvoie à l'ouvrage de Knud Ottosen (1993) qui expose dans son intégralité les différents types d'enchaînement possibles. Je me permets de signaler l'erreur de lecture concernant le V. Ad quam factura tua et non hac hora tui du répons Libera me domine de morte.

5. Albi 15 est aussi le plus ancien témoin pour le verset Anime eorum du R. Requiem eternam ( $1^{\mathrm{er}}$ nocturne). C'est encore le cas du R. Ne perdas me du $3^{\mathrm{e}}$ nocturne.

6. Claustra inferni confracta sunt et monumenta sanctorum aperta sunt, et vox omnium eorum dicit: jam advenisti rex.

7. Braga, Museu da Sé Catedral, Officium defunctorum (XV $\mathrm{XV}^{\mathrm{e}}$ siècle). Le verset Claustra inferni est lié au répons Libera me Domine de morte et non au répons Libera me Domine de viis. Le texte adopte sensiblement les mêmes idées au départ, mais privilégient ensuite la vision paradisiaque et la paix de Dieu plutôt que sa venue et son jugement: Claustra inferni confracta sunt, portae paradisi apertae sunt, sed tu Domine dona eis requiem. Il faut donc nuancer fortement le propos tenu par Ottosen (1993, p. 318) qui a, un peu rapidement, lié Albi 15 et Braga. Le caractère propre de la version de Braga a été souligné par Solange Corbin (1952, p. 277).

8. Les versets ne figurent pas et l'enchaînement incohérent des pièces entre les deux folios montre qu'il manque, à cet endroit, un folio dans le manuscrit.

9. Le V. Plangent est: Plangent se super se omnes tribus terre. Vix justus salvabitur et ego ubi apparebo? Le V. Nunc Christe est: Nunc Christe te deprecor miserere peto qui venisti redimere, perpetim veni salvare. Ce sont ces deux fins indiquées en gras qui sont volontairement interverties comme en témoigne le grattage très visible à cet endroit, sur le manuscrit.

10. Le V. 38 Creator omnium comporte précisément des variantes textuelles qui vont toutes dans ce sens. Au lieu de dire creator omnium... qui nos de limo terre/ corpusque nostrum/ de sepulchro facias resuscitari/ Albi 15 propose creator omnium... qui me de limo terre/ corpusque meum / de seulchro facias me resuscitari.

11. Il manque un des huit versets - Angelorum gaudia - seulement présent dans un des quatre témoins manuscrits connus. 